EPJ Web of Conferences 116, 11013 (2016)

DOI: $10.1051 /$ epjconf/201611611013

(C) Owned by the authors, published by EDP Sciences, 2016

\title{
Opportunities within ASTERICS
}

\author{
Rob van der Meer ${ }^{\mathrm{a}}$ and Giuseppe Cimò for the ASTERICS Consortium \\ ASTRON, Dwingeloo, The Netherlands
}

\begin{abstract}
ASTERICS ${ }^{1}$, The Astronomy ESFRI and Research Infrastructure Cluster project, brings together astronomers and astroparticle physicists of 22 institutes in Europe to help Europe's world-leading observatories work together to find common solutions to their Big Data challenges, their interoperability and scheduling, and their data access, searching for cross-cutting solutions with mutual and wide-ranging benefit to all concerned. ASTERICS is a four year project, funded through the European Union's Horizon 2020 Framework Programme. The facilities supported by ASTERICS include SKA, CTA, KM3NeT, E-ELT. ASTERICS aims to open up multi messenger astronomy to all scientists and the public through the Virtual Observatory and the citizen science work. I will draw a picture of the landscape in which ASTERICS operates and the possible interaction with the Very Large Volume Neutrino Telescope community. Attention will be given to emerging opportunities for the Neutrino community and how these can be recognised or created.
\end{abstract}

\section{Introduction}

The astronomy community and the astroparticle community are building for the future. The European Strategy Forum for Research Infrastructures (ESFRI) Roadmap identifies new Research Infrastructures (RI) of pan-European interest corresponding to the long term needs of the European research communities, covering all scientific areas. At the moment, four large ground based Astronomy and astroparticle physics facilities are on the ESFRI Roadmap 2010 [1] and are being prepared or under construction. Each of these four facilities will be capable of delivering transformational science, moving boundaries in resolution, accumulating surface or volume, or completely new science. During the development and exploration of these facilities, the developers and users will encounter major challenges on data handling, and with the large science and engineering groups they will find solutions to all of these. It should be possible to increase the scientific outcome and decrease the development time by joining forces.

ASTERICS (Astronomy ESFRI and Research Infrastructure Cluster) is a project in the Horizon 2020 Work Programme INFRADEV-4-2014/2015 Call - "Implementation and operation of cross-cutting services and solutions for clusters of ESFRI and other relevant research infrastructure initiatives". ASTERICS brings together for the first time astronomers and astroparticle physicists of 22 institutes from six European countries to help Europe's world-leading observatories work together to find common

\footnotetext{
a e-mail: meer@astron.nl

${ }^{1}$ ASTERICS project website: www. asterics2020.eu
}

This is an Open Access article distributed under the terms of the Creative Commons Attribution License 4.0, which permits unrestricted use, distribution, and reproduction in any medium, provided the original work is properly cited. 
solutions. The project, coordinated by ASTRON, The Netherlands, is funded 15 million Euros over the course of four years, beginning May 1st, 2015.

\section{The ASTERICS project}

\subsection{Objectives}

The focus of ASTERICS is on four ESFRI facilities, each with its own window on the universe:

- SKA, Square Kilometre Array, radio waves

- CTA, Cherenkov Telescope Array, gamma rays

- KM3NeT, Kilometre cube Neutrino Telescope, neutrinos

- E-ELT, European Extremely Large Telescope, visible light.

The main objective of ASTERICS is to join forces. This will result in finding common solutions to data challenges, interoperability and scheduling, and data access, searching for cross-cutting solutions with mutual and wide-ranging benefit to many facilities and user groups, gathering critical mass and creating economy of scale and exploiting complementarities. Besides the above four, ASTERICS also has close links to facilities that want to be on the ESFRI roadmap, like the Einstein Telescope (ET). Since no data is coming out of the focus facilities yet, the data from complementary, pathfinder and precursor facilities, like LOFAR, Euclid, LSST, VIRGO, LIGO, eVLBI, HESS, MAGIC, ANTARES, IceCube will be used for comparing data formats, design new data structures, and analysis software, benchmark hardware, and test prototypes. ASTERICS aims to open up multi messenger astronomy to all scientists and the public through the Virtual Observatory (VO), as described in Sect. 2.3.4, and citizen science work, as described in Sect. 2.3.2, where members of the general public are encouraged to undertaken scientific work in collaboration with or under the direction of professional scientists and scientific institutions. Following from these objectives, there will be plenty of opportunities for project partners and linked facilities to contribute to the search for common solutions and to profit from solutions from other fields. The project results will be widely distributed. It is in the interest of all scientific communities to actively participate and recognise, or create, emerging opportunities.

\subsection{Added value}

The ESFRI facilities are very well capable of producing interesting science results. However, the added value of ASTERICS will be to create the capability to move the science frontiers beyond the expectations within the science cases of the facilities, and lift the outcome far above the results of the single facilities. To raise the single ESFRI facilities to the level of intended added value, ASTERICS will start with coordinating the individual requirements, to disseminate results, educate and train the developers, manager and users of facilities. The close and direct involvement of ESFRI project and pathfinder staff is essential in realising the major ASTERICS ambitions. There are several opportunities for the VLVnT community to work together with the other facilities to exchange information and to showcase their knowledge and experience with the precursor experiments. The intended added value of ASTERICS compared to the individual ESFRI facilities is summarised in paragraphs below.

\subsubsection{Design requirements}

The ESFRI facilities will have their own design requirements aimed for optimal science return of the own facility. 
ASTERICS aims at:

- enabling interoperability between facilities

- minimising fragmentation of research developments, stimulating collaboration between or mergers of several small groups working on parts of the research of a much larger challenge, into larger (virtual) teams

- encouraging cross-fertilisation, where ideas of one research groups inspires another research group to find solutions for unrelated issues

- developing joint multi-wavelength/multi-messenger capabilities

- opening-up the next generation of observatories to discovery and usage (and re-usage) of data and data reduction and analysis software

- searching for generic approach to the data challenge.

\subsubsection{Technology choices}

The ESFRI facilities will (have to) go for mature technology choices at some decision points.

ASTERICS aims at:

- identifying areas of rapid technology development

- creating complementary and synergic approaches across the project

- finding significant added value.

\subsubsection{Observations}

The ESFRI facilities will optimise their own observations. They will have exceptional science cases per facility.

ASTERICS aims at:

- coordinating and harmonising joint and efficient scheduling, operation and interoperability

- multi-wavelength / multi-messenger observations

- engaging with society

- enlarging the Citizen science capacity and usage

- achieving the Science 2.0 goals (open access to all data, analysis tools, publication platforms).

\subsubsection{Data analysis}

The ESFRI facilities and their users will focus on the analysis of their own data.

ASTERICS aims at:

- data integration across ESFRI facilities

- open standards

- adapting Virtual Observatory framework and tools

- e-science infrastructures

- data mining

- co-development for software and hardware

- software libraries.

\subsection{Organisation}

The activities within ASTERICS are organised in five work packages. The starting points are the data challenges. There are activities around data collection at the facilities, data access for users, standardisation for data and meta data, interoperability, usability of analysis tools, re-use of data and software, awareness increase of users, general public, and policy makers. Besides reaching out to research communities like the VLVnT community at their conferences and meetings, also interest groups 
are targeted, e.g. during the Astronomical Data Analysis Software \& Systems (ADASS) conference by F. Pasian [5]. The specific tasks and activities of the five ASTERICS work packages are described in the following paragraphs.

\subsubsection{WP1: ASTERICS Management Support Team (AMST)}

The ASTERICS Management Support Team (AMST) facilitates the project organisation and governance. It coordinates the communication between internal and external stakeholders and coordinates the work to be done in the project. The project is built on four ESFRI facilities originating from four completely different science disciplines that have developed their fields separately since their origin (optical astronomy, gamma ray physics, neutrino physics, and radio astronomy). One major task of the AMST is to morph these four pillars of the project into one big family, while conserving the individual characteristics of the science disciplines and to accelerate the progress by enthusiastic collaboration.

\subsubsection{WP2: Dissemination, Engagement and Citizen Science (DECS)}

The first task of WP2 is to disseminate the project results to the stakeholders and to promote ASTERICS and the facilities it serves to as large an audience as possible, by creating high quality branding and promotional outreach material. Possible audiences are: scientific and technical communities, academia, private industry, other public research centres, SME's, policy makers, general public. The second task is to engage the public in mass participation events through the Citizen Science platform. This platform serves two purposes: entertaining and educating the general public in an (inter)active way, and giving the scientist a flexible tool by using a much larger number of eyes and brains. To make optimal use of the Citizen Science platform, scientist should ask themselves: "What could I do with a million eyes, to improve my scientific return on the data I have collected?" To develop relevant questions for the VLVnT community, researchers are invited to participate in the citizen science brainstorming workshops.

\subsubsection{WP3: Observatory E-environments Linked by common ChallengeS (OBELICS)}

The work package OBELICS (Observatory E-environments Linked by common ChallengeS) aims at co-development of the robust, scalable, flexible handling and exploitation of huge data streams and distributed petascale database systems. The work package aims to enable interoperability and software re-use in the three fields of data generation, integration, and analysis. The work package is divided into three tasks, each focussing on one of these fields.

\subsubsection{WP4: Data Access, Discovery and Interoperability (DADI)}

DADI (Data Access, Discovery and Interoperability) is a prolongation of the Virtual Observatory (VO) projects that started fifteen years ago, and in particular of the series of Euro-VO projects supported by the European Commission (Genova et al., 2015, [2]). The goal is to make the ESFRI and pathfinder projects data available for discovery and usage by the whole astronomical community, interoperable in a homogeneous international framework, and accessible with a set of common tools. In order to optimise evolving the VO, the work is organised around a sequence of actions: train and support ESFRI and pathfinder staff, collect requirements and feedback from the users, adapt the VO framework to the collected requirements and train again.

The DADI work to optimize usage of the data from large projects through the VO is in line with the priority of the IVOA $^{2}$ (International Virtual Observatory Alliance). The VO is a framework

\footnotetext{
2 http: //www.ivoa.net
} 
that allows data services following common rules, using common tools. ASTERICS wants to support implementation of the IVOA framework in the ESFRI projects and their pathfinders and to contribute to the development of the VO standards and tools as well. The VO is the interface between the domain specific and generic infrastructure and it works on new and archive data. This was demonstrated in a visualisation of IceCube-40 string data with VO tools by Abbasi et al. [3].

During the ASTERICS project there are recurring events where the partner projects and interested scientists can interact to improve the $\mathrm{VO}$ and their own data analysis. These events are: the DADI technology Forums, to inform about technological developments and build collaborations, the annual ASTERICS European Schools (with open call to European early career scientists, Post-doc, 50 participants), the two ESFRI Forum and training events (network, share lessons learnt, discuss requirements, training), and the two Data Provider Forums (open to all European Data providers). These meetings are useful also for the VLVnT community by providing input for optimising the $\mathrm{VO}$ and for learning how to use the $\mathrm{VO}$ tools.

\subsubsection{WP5: CLEOPATRA (Connecting Locations of ESFRI Observatories and Partners in Astronomy for Timing and Real-time Alerts)}

CLEOPATRA (Connecting Locations of ESFRI Observatories and Partners in Astronomy for Timing and Real-time Alerts) aims specifically at synergetic observing modes, and fast and reliable access to large data streams. Tasks in the work package are: technology development for fibre connectors, relaying alerts, data streaming software, data dissemination, and advanced scheduling algorithms. This builds on WRE (White Rabbit Ethernet) and the EC EXPReS/NEXPRES projects. The scheduling, triggering and timing can be of interest to the VLVnT community for the Astrophysical Multimessenger Observatory Network (AMON), presented at this VLVnT workshop by Douglas Cowen [4].

\section{Conclusion}

The close and direct involvement of ESFRI project and pathfinder staff is essential in realising the major ASTERICS ambitions. There are several opportunities for the VLVnT community to work together with the other facilities to exchange information and to showcase their knowledge and experience with the precursor experiments. All work packages have meetings to contact users and projects for input/output. Everyone is encouraged to use these meetings to engage with the project and to maximise the output to the community. Keep an eye on www . asterics2020. eu for the schedule of meetings.

STERICS is a project funded by the European Commission under the Horizon2020 programme (id 653477). All the partner institutions and individual participants are gratefully acknowledged for their work in the project.

\section{References}

[1] ESFRI Roadmap 2010, http: //www . esfri.eu/roadmap-archive (2010)

[2] F. Genova et al., arXiv:1506.06567, DOI: 10.1016/j.ascom.2015.03.001, Astronomy and Computing 11, $181(2015)$

[3] R. Abbasi et al., arXiv:1104.5187, Phys. Rev. D 84, 082001 (2011)

[4] D. Cowen et al., this proceedings (EDP Sciences, to be published)

[5] F. Pasian, M.A. Garrett, F. Genova, G. Lamanna, S. Serjeant, A. Szomoru, R. van der Meer, Astronomical Data Analysis Software and Systems XXV, ASP Conference Series, in press, 2016 